\title{
Relação entre os preços dos alimentos e das commodities agropecuárias
}

\author{
Kellen Cristina Campos Fernandes" \\ Reginaldo Santana Figueiredo**
}

\begin{abstract}
Resumo
O objetivo do presente trabalho foi verificar se há relacionamento de longo prazo e transmissão de preços entre os mercados de commodities agropecuárias e os alimentos da cesta básica. Para atingir os objetivos da pesquisa, que envolve a análise da transmissão de preços nos mercados de alimentos e commodities, inicialmente, buscou-se testar a estacionariedade das séries em estudo. Posteriormente, utilizou-se o teste de cointegração de Engle e Granger e o teste de cointegração de Johansen. Para analisar se há uma relação causal entre as séries estudadas, foi realizado o teste de causalidade de Granger. Verificouse que existe relacionamento de longo prazo entre os preços dos alimentos e das commodities agropecuárias, de forma que as variações nos preços das commodities podem afetar os preços dos alimentos.
\end{abstract}

Palavras-chave: Cointegração. Transmissão de preços. Commodities. Alimentos.

* Doutoranda em Agronegócio, Universidade Federal de Goiás (UFG). E-mail: kellenccfernandes@ gmail.com

** Pós-doutorado em Modelagem e Simulação no departamento de Engenharia Industrial na Texas A\&M University e professor da Escola de Agronomia, Universidade Federal de Goiás (UFG). E-mail: emaildesantana@gmail.com

http://dx.doi.org/10.5335/rtee.v26i54.12121

Submissão: 10/12/2020. Aceite: 19/04/2021.

Teoria e Evidência Econômica - a. 26, n. 54, p. 115-131, jan./jun. 2020 


\section{Introdução}

As preocupações com a volatilidade dos preços dos alimentos e o aumento dos picos de preços aumentaram depois da crise de preços de 2007-2008, momento em que houve aumento da fome e da pobreza de populações já carentes. A crise refletiu sobre os mercados mundiais de grãos, que naquela época não respondiam às variações de oferta e demanda e aos custos de produção, mas sim às variações dos mercados financeiros (TORRES, 2017).

Gilbert (2010) evidencia que existe um conjunto de fatores que leva ao aumento dos preços dos alimentos, os quais incluem a demanda por biocombustíveis, a especulação nos mercados de commodities, as políticas agressivas de estoques dos países, as restrições comerciais, os choques macroeconômicos na oferta de moeda, as taxas de câmbio e o crescimento econômico da China.

Tadasse et al. (2014) estudaram o aumento dos preços internacionais das commodities. Segundo os autores, em 2007-2008, os preços nominais de quase todas as commodities alimentares aumentaram em mais de 50\%. Três anos após os picos globais dos preços dos alimentos em 2007-2008, os preços dos alimentos dispararam novamente em 2010-2011. Embora os dois eventos tenham sido diferentes em termos das commodities afetadas, a forte correlação foi encontrada entre a maioria dos preços dos alimentos.

A volatilidade de preços das commodities agrícolas tem se tornado objeto de estudo de vários pesquisadores, na busca da compreensão dos impactos que os riscos de preços podem causar, principalmente, sobre a segurança alimentar. Assim, a discussão desse tema é de suma importância para o Brasil, dada a sua relevância como grande produtor de alimentos, uma vez que o país poderá ser afetado, positiva ou negativamente, pelas grandes volatilidades de preços.

Acredita-se que o grande agronegócio, voltado para a produção de commodities e biocombustíveis, afeta a produção de alimentos. Em virtude da escassez de alimentos em alguns períodos, muitas vezes oriunda de problemas climáticos e de problemas relacionados ao acesso aos alimentos pela população, há um sentimento presente na sociedade de que a produção de alimentos está enfrentando a concorrência pelo uso das terras, onde, entre outros fins, inclui-se a produção de biocombustíveis e de demais culturas voltadas à exportação. Desta forma, em alguns momentos esta produção pode representar potenciais conflitos com os 
propósitos da segurança alimentar, porém, ao mesmo tempo, pode oferecer novas oportunidades nas zonas rurais.

Apesar de muitos estudos analisarem a relação entre os mercados de combustíveis e biocombustíveis e os mercados de alimentos, poucos estudos contemplaram a relação entre os mercados de commodities agrícolas e os mercados de alimentos. Nesse contexto, o objetivo da presente pesquisa é verificar se há relacionamento de longo prazo e transmissão de preços das commodities para os preços dos alimentos no estado de Goiás.

\section{Referencial teórico}

Tradicionalmente, colheitas, estoques e renda eram considerados os principais determinantes dos preços dos alimentos. Recentemente, surgiram novos impulsionadores que estão enraizados em uma integração mais próxima dos mercados de alimentos com os mercados financeiro, de commodities e de energia, despertando o interesse dos pesquisadores e a realização de novas pesquisas.

A relação entre os mercados de combustíveis e biocombustíveis e os mercados de alimentos tem sido objeto de estudo de diversos autores (TADASSE et al., 2014; NICOLA; PACE; HERNANDEZ, 2016; ABDLAZIZ; RAHIM; ADAMU, 2016; AKÉ, 2017; AL- MAADID et al., 2017; FILIP et al., 2017; PAL; MITRA, 2017, 2018; JAWAD et al., 2018; FASANYA; AKINBOWALE, 2019).

Nicola, Pace e Hernandez (2016) constataram que os retornos de preços de energia e commodities agrícolas são altamente correlacionados, especialmente nos casos do milho e do óleo de soja, que são insumos importantes na produção de biocombustíveis. Observou-se que a volatilidade do mercado de ações está positivamente associada ao movimento de retornos de preços entre os mercados, especialmente após a crise de 2007.

Seguindo essa mesma tendência, Al-Maadid et al. (2017) também examinaram as relações entre os preços de alimentos e energia e verificaram a existência de relações significativas entre os preços dos alimentos e do petróleo e do etanol. Além disso, os autores destacam a contribuição da crise alimentar de 2006 e da crise financeira de 2008 para as mudanças mais significativas nos transbordamentos de risco entre as séries de preços consideradas.

Pal e Mitra (2018) analisaram se a interdependência entre o preço do petróleo bruto e os preços mundiais de alimentos diferiu significativamente nos períodos 
pré-crise, durante a crise e pós-crise. Os autores identificaram uma forte interdependência positiva entre o preço do petróleo bruto e o índice mundial de preços de alimentos. Essa constatação indica que, com o aumento do preço do petróleo bruto, mais grãos serão desviados para a geração de biocombustíveis, o que pode acarretar um grave problema de insegurança alimentar nos países em desenvolvimento, dado que as pessoas de baixa renda apresentam uma maior propensão a consumir e uma porção maior da sua cesta de consumo é feita de alimentos, mais especificamente cereais.

Já os dados das séries de preços analisadas no trabalho de Filip et al. (2017) apontam que os biocombustíveis não podem ser considerados como principal fonte de altos preços dos alimentos e, consequentemente, da escassez de alimentos. No entanto, o estudo mostra que, em mercados específicos e em períodos específicos, existem transmissões de preços estatisticamente significativas entre as commodities agrícolas e os combustíveis, que foram possibilitadas pelas políticas governamentais de incentivo à produção de biocombustíveis. Os autores concluem que a transmissão de preços nesses mercados não é puramente baseada em tecnologia, inclui também a influência dos mercados financeiros.

Tadasse et al. (2014) identificaram ligações crescentes entre os mercados de alimentos, energia e financeiro, as quais explicam grande parte dos picos de volatilidade observados nos preços dos alimentos. Os autores afirmam que a especulação financeira amplifica os picos de preços no curto prazo e a volatilidade dos preços do petróleo impulsiona a volatilidade dos preços no médio prazo.

A influência do preço do petróleo, enquanto fator econômico global, sobre o comportamento dos preços de commodities agrícolas, como trigo, milho, soja e arroz, sob cenários adversos e prósperos de mercado foi tema de estudo de Jawad et al. (2018). Foram encontradas evidências de simetria na dependência da cauda entre variáveis e de assimetria nas repercussões do petróleo para commodities agrícolas que se intensificam durante a turbulência financeira.

Aké (2017) identificou a existência de uma relação não linear entre os retornos dos preços dos alimentos, o uso de biocombustíveis, a atividade econômica e os preços da energia. Para o autor, a atividade econômica global que utiliza energia provoca aumento dos preços dos combustíveis e dos alimentos. Quando o preço dos combustíveis aumenta, torna-se rentável a produção de biocombustíveis. A expansão econômica, segundo o autor, também faz com que uma parcela maior da população 
consuma mais bens básicos, tornando o alimento mais caro devido à demanda crescente e ao aumento nos custos de produção, como transporte e fertilizantes.

Fasanya e Akinbowale (2019) analisaram os transbordamentos de retorno e volatilidade do petróleo bruto e dos preços dos alimentos na Nigéria. Os autores identificaram a existência de evidências de interdependência entre os preços do petróleo bruto e os preços dos alimentos com base nos índices de transbordamento. O estudo realizado por Abdlaziz, Rahim e Adamu (2016) também evidenciou a existência de cointegração entre os preços dos alimentos, a taxa de crescimento do produto interno bruto e os preços do petróleo.

Pal e Mitra (2017) encontraram uma relação de cointegração, estatisticamente significativa, entre os preços do petróleo bruto e os índices de preços dos alimentos e suas subcategorias, tais como laticínios, cereais, óleo vegetal e açúcar. Os preços mundiais dos alimentos, juntamente com os preços de cereais, óleos vegetais e açúcar, acompanham e são conduzidos pelos preços do petróleo bruto, resultados que permanecem relevantes da perspectiva da política de curto prazo e para o transbordamento das mudanças nos preços do petróleo bruto para o índice mundial de preços de alimentos em longo prazo.

Os estudos de Rezende, Oliveira Neto e Silva (2018) e de Ceballos et al. (2017) analisaram a volatilidade e a transmissão do preço dos mercados de commodities agrícolas internacionais para os mercados de alimentos domésticos. Rezende, Oliveira Neto, Silva (2018) analisaram a volatilidade e a transmissão do preço do trigo internacional para os preços domésticos desse grão e seus derivados no Brasil. Verificou-se que a variação de $1 \%$ no preço do trigo norte-americano ampliaria em $1,29 \%$ a variação do preço da farinha de trigo no Brasil.

Os derivados do trigo brasileiro (farinha de trigo e pão francês) sofrem maior influência das variações ocorridas nos preços do trigo norte-americano.

Ceballos et al. (2017) verificaram a transmissão de preços e a volatilidade de curto prazo das principais commodities de grãos para 41 produtos alimentícios domésticos em 27 países da África, da América Latina e do Sul da Ásia. Em termos de transmissão de preços, observou-se interações significativas de mercados internacionais para mercados domésticos em poucos casos. Para calcular os transbordamentos de volatilidade, simulou-se um choque equivalente a um aumento de $1 \%$ na volatilidade condicional dos retornos de preços no mercado internacional e avaliou-se o efeito sobre a volatilidade condicional dos retornos de preços no mercado doméstico. A transmissão da volatilidade foi estatisticamente significativa em 
apenas um quarto dos mercados de milho testados, mais da metade dos mercados de arroz testados e todos os mercados de trigo testados.

\section{Procedimentos metodológicos}

Para atingir os objetivos propostos pela presente pesquisa, foram utilizados métodos econométricos aplicados às séries temporais, uma vez que permitem a análise do relacionamento de curto e de longo prazos entre as variáveis.

Foram utilizados dados secundários de séries históricas mensais de preços médios do arroz $(\mathrm{R} \$ / 5 \mathrm{~kg})$, feijão comum cores $(\mathrm{R} \$ / \mathrm{kg})$, leite de vaca longa vida integral $(\mathrm{R} \$ / \mathrm{l})$, milho $(\mathrm{R} \$ / \mathrm{sc})$, óleo de soja $(\mathrm{R} \$ / 900 \mathrm{ml})$, soja $(\mathrm{R} \$ / \mathrm{sc})$ e sorgo ( $\mathrm{R} \$ / \mathrm{sc})$, no estado de Goiás, entre os meses de janeiro de 2005 e abril de 2019, divulgados nas bases de dados da Conab e do Instituto Mauro Borges (IMB).

As séries de preços das commodities consistem em milho, soja e sorgo, que são os principais produtos agropecuários produzidos em Goiás. Os demais produtos serão considerados como alimentos, visto que estes fazem parte da cesta básica. Após o levantamento das séries de preços, foram aplicados os testes de raiz unitária, para verificar a estacionariedade e identificar a ordem de integração das séries analisadas. Os dados foram tabulados e processados no programa Microsoft Excel 365. Posteriormente, para o cálculo dos demais testes, foi utilizado o Eviews, versão 9.

O primeiro passo foi verificar se as séries eram estacionárias. Uma série temporal é considerada estacionária, se suas propriedades estatísticas não variam em relação ao tempo (PINDYCK; RUBINFELD, 2004). Portanto, as séries estacionárias têm a média e a variância constantes ao longo do tempo, e a covariância entre os valores defasados das séries depende apenas da defasagem entre eles e não do tempo (GUJARATI; PORTER, 2011).

Um teste muito usual para detectar a estacionariedade de uma série é o teste de raiz unitária. Um dos testes pioneiros para a verificação da existência de raiz unitária foi desenvolvido por Dickey e Fuller (1979) e é conhecido como teste DF.

Bueno (2011) argumenta que um dos problemas referentes ao teste DF é que ele considera o termo de erro como um ruído branco, podendo ser utilizado somente para as séries em que os erros não são autocorrelacionados, o que limita o poder do teste. Dickey e Fuller desenvolveram outro teste, o teste de Dickey e Fuller Aumentado $(\mathrm{ADF})$, incluindo defasagens em relação à variável que está sendo analisada.

Teoria e Evidência Econômica - a. 26, n. 54, p. 327-348, jan./jun. 2020 
A hipótese nula do teste $\mathrm{ADF}$ é a mesma do teste $\mathrm{DF}$. $\mathrm{O}$ teste $\mathrm{ADF}$ é realizado utilizando os mesmos valores críticos de Dickey e Fuller, desde que se corrija o modelo, considerando as demais variáveis defasadas, os valores críticos permanecem inalterados (BUENO, 2011). A principal vantagem do teste $\mathrm{ADF}$ em relação ao DF é que, ao incluir um número suficiente de defasagens, garante-se que os resíduos sejam autocorrelacionados.

Pindyck e Rubinfelf (2004) afirmam que grande parte das séries temporais econômicas não é gerada por processos estacionários. Estimar uma regressão de duas séries temporais não estacionárias pode levar a resultados espúrios, no sentido de que testes de significância convencionais tenderão a indicar uma relação entre as variáveis quando de fato ela não existe. Algumas vezes, duas variáveis podem ser não estacionárias, mas uma combinação linear dessas variáveis pode ser estacionária.

Hill, Griffiths e Judge (2010) exemplificam que se yt e xt são variáveis não estacionárias de ordem um, sua diferença ou qualquer combinação linear delas pode ser estacionária. Nesse caso, pode-se verificar que yt e xt são consideradas cointegradas. Duas variáveis são cointegradas se tiverem uma relação de equilíbrio ou de longo prazo entre elas.

Cointegração, portanto, indica que yt e xt compartilham tendências estocásticas semelhantes e, de fato, como sua diferença et é estacionária, elas não divergem muito uma da outra e apresentam uma relação de equilíbrio de longo prazo. No curto prazo, há desvios dessa tendência comum, de modo que et é o erro de equilíbrio, que representa desvios a partir da relação de longo prazo (HILL; GRIFFITHS; JUDGE, 2010). Esse conceito de cointegração recebeu um tratamento formal em Engle e Granger (1987), tornando as regressões que envolvem variáveis integradas de ordem um potencialmente significativas.

Gujarati e Porter (2011) explicam que existem vários métodos para se testar a cointegração e afirmam que um dos métodos mais simples é realizar o teste de raiz unitária de Dickey-Fuller ou Dickey-Fuller Aumentado para verificar a estacionariedade dos resíduos da regressão de cointegração.

O teste de cointegração de Engle e Granger (1987) é indicado para ser realizado em uma única equação. Em um modelo de várias variáveis, é possível existir mais de um vetor de cointegração. Especificar quais equações devem ser testadas constitui um problema a ser resolvido antes do teste. Não obstante, a solução estará na especificação das relações econômicas entre as variáveis (BUENO, 2011). 
Para atingir os objetivos da pesquisa, que envolve a análise do relacionamento de longo prazo entre os mercados de alimentos e commodities, foi analisado, através do teste de cointegração de Johansen (1988), em um contexto multivariado, onde existe a possibilidade de mais de um vetor de cointegração estar presente. Esse teste segue os mesmos princípios que a abordagem de Engle-Granger para a cointegração, na medida em que a ordem de integração das variáveis é primeiro avaliada, se as variáveis forem I(1), o procedimento de Máxima Verossimilhança de Johansen pode ser usado para determinar se existe um relacionamento de equilíbrio de longo prazo entre as variáveis.

O procedimento de Johansen produz um conjunto de resultados que podem ser usados para determinar o número de vetores de cointegração presentes. Haverá um possível vetor $g$-1, em que $g$ é o número de variáveis incluídas no modelo. Com base nesse resultado, os coeficientes de longo prazo podem ser determinados e o modelo de correção de erros resultante é produzido. Esta abordagem produz dois conjuntos de coeficientes, os coeficientes de longo prazo $(\square)$ e $(\square)$, que correspondem às velocidades dos coeficientes de ajuste e, aproximadamente, equivalem ao termo de correção de erro.

Em seguida, foi realizado o teste de Causalidade de Granger, que avalia a eventual procedência entre essas séries, um relacionamento de curto prazo. O teste de Causalidade de Granger apresenta uma noção limitada na qual os valores passados de uma série são úteis para prever valores futuros de outra série (WOOLDRIDGE, 2013). Stock e Watson (2004) explicam que a causalidade de Granger significa que, se $\mathrm{X}$ causa $\mathrm{Y}$ no sentido de Granger, então $\mathrm{X}$ é um previsor útil de $\mathrm{Y}$, dadas as outras variáveis da regressão.

\section{Resultados e discussão}

Inicialmente, buscou-se analisar o comportamento individual das séries de preços dos alimentos e das principais commodities produzidas no estado de Goiás, visando identificar o comportamento das séries de preços estudadas. Na Tabela 1, são apresentadas as estatísticas descritivas dos preços médios mensais de: arroz, feijão, leite, milho, óleo de soja, soja e sorgo no estado de Goiás, em que é possível verificar as medidas de tendência central, dispersão e distribuição. 
Tabela 1 - Estatísticas descritivas

\begin{tabular}{l|r|r|r|r|r|r|r}
\hline \multicolumn{1}{c|}{ Estatísticas } & Arroz & Feijão & Leite & Milho & $\begin{array}{c}\text { Óleo de } \\
\text { soja }\end{array}$ & \multicolumn{1}{c|}{ Soja } & \multicolumn{1}{c}{ Sorgo } \\
\hline Média & 9,79 & 4,39 & 1,98 & 22,22 & 2,87 & 48,57 & 16,65 \\
Mediana & 9,53 & 4,20 & 1,77 & 21,97 & 2,91 & 44,78 & 16,36 \\
Máximo & 14,93 & 12,24 & 3,86 & 43,06 & 4,11 & 81,71 & 31,17 \\
Mínimo & 5,56 & 2,09 & 1,03 & 12,73 & 1,96 & 20,90 & 9,74 \\
Desvio padrão & 2,57 & 1,70 & 0,69 & 6,55 & 0,49 & 16,30 & 4,54 \\
Coeficiente de variação & $26,25 \%$ & $38,72 \%$ & $34,85 \%$ & $29,48 \%$ & $17,07 \%$ & $33,56 \%$ & $27,27 \%$ \\
Assimetria & 0,13 & 2,15 & 0,53 & 0,83 & 0,00 & 0,02 & 0,73 \\
Curtose & 1,95 & 9,09 & 2,44 & 3,59 & 2,35 & 1,84 & 3,42 \\
Jarque-Bera & 8,37 & 395,14 & 10,22 & 22,07 & 3,05 & 9,54 & 16,63 \\
Probabilidade & 0,02 & 0,00 & 0,01 & 0,00 & 0,22 & 0,01 & 0,00 \\
\hline
\end{tabular}

Fonte: dados da pesquisa, 2020.

Analisando os preços médios mensais verificados no período de janeiro de 2005 a abril de 2019, dispostos na Tabela 1, observa-se que, dentre os alimentos considerados na análise, o arroz apresentou a maior média de preços $(9,79)$.

Para uma análise comparativa, a Tabela 1 também apresenta os coeficientes de variação dos produtos considerados na presente pesquisa. Comparando os coeficientes de variação dos preços dos alimentos, observa-se que o óleo de soja é o produto com menor volatilidade, visto que a dispersão relativa, medida pelo coeficiente de variação $(17,07 \%)$, é inferior aos demais. O alimento que apresentou a maior volatilidade foi o feijão, com coeficiente de variação de $38,72 \%$. Em relação às commodities, o sorgo apresentou a menor volatilidade $(27,27 \%)$ e a soja, a maior volatilidade $(33,56 \%)$.

Para verificar a normalidade da distribuição dos dados, utilizou-se o teste de Jarque- Bera e os valores estimados para a assimetria e curtose, que indicaram que a série de preços de óleo de soja se aproxima da distribuição normal. Já as demais séries mostraram que os dados não apresentam distribuição normal, ao rejeitar a hipótese nula de normalidade.

A correlação linear entre preços médios mensais de arroz, feijão, leite, milho, óleo de soja, soja e sorgo no estado de Goiás é apresentada na Tabela 2. 
Tabela 2 - Matriz de correlação

\begin{tabular}{|c|c|c|c|c|c|c|c|}
\hline $\begin{array}{c}\text { Correlação } \\
\text { Estatística-t } \\
\text { p-valor }\end{array}$ & Arroz & Feijão & Leite & Milho & $\begin{array}{c}\text { Óleo de } \\
\text { soja }\end{array}$ & Soja & Sorgo \\
\hline \multirow[t]{2}{*}{ Arroz } & $\begin{array}{l}1,000 \\
----\end{array}$ & & & & & & \\
\hline & 0,935 & & & & & & \\
\hline \multirow[t]{3}{*}{ Feijão } & 12,483 & ----- & & & & & \\
\hline & 0,000 & ----- & & & & & \\
\hline & $\begin{array}{l}0,919 \\
30,354\end{array}$ & 0,685 & 1,000 & & & & \\
\hline \multirow{2}{*}{ Leite } & $\begin{array}{l}30,354 \\
0,000\end{array}$ & $\begin{array}{l}12,216 \\
0,000\end{array}$ & ---- & & & & \\
\hline & 0,734 & 0,746 & 0,731 & 1,000 & & & \\
\hline \multirow[t]{3}{*}{ Milho } & 14,044 & 14,572 & 13,941 & ----- & & & \\
\hline & 0,000 & 0,000 & 0,000 & ----- & & & \\
\hline & 0,824 & 0,599 & 0,726 & 0,800 & 1,000 & & \\
\hline \multirow[t]{3}{*}{ Óleo de soja } & 18,934 & 9,734 & 13,740 & 17,304 & ---- & & \\
\hline & 0,000 & 0,000 & 0,000 & 0,000 & ----- & & \\
\hline & 0,939 & 0,623 & 0,953 & 0,697 & 0,764 & & \\
\hline \multirow[t]{3}{*}{ Soja } & 30,818 & 10,169 & 29,269 & 16,114 & 20,667 & ----- & \\
\hline & 0,000 & 0,000 & 0,000 & 0,000 & 0,000 & ----- & \\
\hline & 0,724 & 0,743 & 0,758 & 0,906 & 0,741 & 0,798 & 1,000 \\
\hline \multirow[t]{2}{*}{ Sorgo } & 13,656 & 14,443 & 15,124 & 27,752 & 14,360 & 17,200 & ----- \\
\hline & 0,000 & 0,000 & 0,000 & 0,000 & 0,000 & 0,000 & ----- \\
\hline
\end{tabular}

Fonte: dados da pesquisa, 2020.

A partir dos resultados apresentados na Tabela 2, verifica-se que todas as séries de preços apresentam correlação positiva entre si.

Para testar a estacionariedade das séries em estudo, aplicou-se o teste de raiz unitária de Dickey-Fuller Aumentado (ADF). A Tabela 3 apresenta os resultados do teste de raiz unitária de Dickey-Fuller Aumentado, em nível para os preços históricos mensais dos produtos considerados na presente pesquisa. 
Tabela 3 - Teste de Raiz Unitária (Dickey-Fuller Aumentado)

\begin{tabular}{l|l|c|c|c}
\hline Série de preços & \multicolumn{1}{|c|}{ Equação ADF } & Estatística ADF & p-valor & Durbin-Watson \\
\hline \multirow{2}{*}{ Arroz } & Em nível, com intercepto & $-1,2557^{n+}$ & 0,6495 & 1,9220 \\
& Em primeira diferença, com intercepto & $-8,7850^{* * *}$ & 0,0000 & 1,9154 \\
\hline \multirow{2}{*}{ Feijão } & Em nível, com intercepto & $-3,1436^{* *}$ & 0,0253 & 1,9348 \\
& Em primeira diferença, com intercepto & $-8,7391^{* * *}$ & 0,0000 & 1,8869 \\
\hline \multirow{2}{*}{ Leite } & Em nível, com intercepto & $-1,1558^{\text {ns }}$ & 0,6928 & 1,9707 \\
& Em primeira diferença, com intercepto & $-9,3993^{* * *}$ & 0,0000 & 1,9769 \\
\hline \multirow{2}{*}{ Milho } & Em nível, com intercepto & $-2,4704^{\text {ns }}$ & 0,1246 & 2,0309 \\
& Em primeira diferença, com intercepto & $-7,8708^{* * *}$ & 0,0000 & 1,9961 \\
\hline \multirow{2}{*}{ Óleo de soja } & Em nível, com intercepto & $-2,2883^{\text {ns }}$ & 0,1770 & 2,0284 \\
& Em primeira diferença, com intercepto & $-8,3567^{* * *}$ & 0,0000 & 1,9990 \\
\hline \multirow{2}{*}{ Soja } & Em nível, com intercepto & $-1,4353^{\text {ns }}$ & 0,5639 & 1,9241 \\
& Em primeira diferença, com intercepto & $-9,2283^{* * *}$ & 0,0000 & 1,9199 \\
\hline \multirow{2}{*}{ Sorgo } & Em nível, com intercepto & $-2,5173^{\text {ns }}$ & 0,1131 & 1,9519 \\
& Em primeira diferença, com intercepto & $-9,9349^{* * *}$ & 0,0000 & 1,9345 \\
\hline
\end{tabular}

*** Estatisticamente significante ao nível de 1\%; ** Estatisticamente significante ao nível de $5 \%$; * Estatisticamente significante ao nível de 10\%; ns: Estatisticamente não significativo.

Fonte: dados da pesquisa, 2020.

De acordo com os resultados da Tabela 3, os resultados do teste ADF para as séries médias mensais de arroz, leite, milho, óleo de soja, soja e sorgo, em nível, com intercepto, o valor da estatística $t$ apresentou resultados superiores aos valores críticos aos níveis de $1 \%, 5 \%$ e $10 \%$. Portanto, não se pode rejeitar a hipótese nula de existência de raiz unitária, em todos os níveis de significância, indicando que todas as séries são não estacionárias em nível. Porém, ao se testar a estacionariedade da série de preços do feijão, em nível, com intercepto, os resultados do teste ADF evidenciaram que a série é estacionária no nível de significância de 5\%, ou seja, pode-se rejeitar a hipótese nula de existência de raiz unitária.

Posteriormente, verificou-se que todas as séries não possuem raiz unitária na primeira diferença, ou seja, quando são transformadas em retornos, as séries são estacionárias. Os valores da estatística de Durbin-Watson em todas as séries apresentaram valores próximos de 2,0, evidenciando que o teste é conclusivo, visto que as séries não apresentam problema de autocorrelação serial.

Para testar a existência de cointegração entre as séries, utilizou-se o teste de cointegração de Engle e Granger, estimando-se uma regressão, pelo Método dos Mínimos Quadrados Ordinários, em que cada alimento é a variável dependente e cada commodity é a variável independente, com o intuito de verificar as relações existentes entre as séries. Em seguida, testou-se a estacionariedade dos resíduos desta regressão por meio do teste $\mathrm{ADF}$ (Tabela 4). 
Tabela 4 - Teste nos resíduos das estimações em pares - Engle-Granger

\begin{tabular}{l|c|c|c}
\multicolumn{1}{c|}{ Pares } & Estatística ADF & p-valor & Durbin-Watson \\
\hline Arroz-milho & $-2,9538^{* *}$ & 0,0415 & 1,9609 \\
Arroz-soja & $-3,9544^{* *}$ & 0,0021 & 1,9051 \\
Arroz-sorgo & $-2,9768^{* *}$ & 0,0392 & 1,9799 \\
\hline Feijão-milho & $-5,1592^{* * *}$ & 0,0000 & 1,9495 \\
Feijão-soja & $-4,3113^{* * *}$ & 0,0006 & 2,0304 \\
Feijão-sorgo & $-4,4242^{* * *}$ & 0,0004 & 1,99631 \\
\hline Leite-milho & $-3,8978^{* * *}$ & 0,0026 & 1,8580 \\
Leite-soja & $-4,6512^{* * *}$ & 0,0002 & 1,9866 \\
Leite-sorgo & $-3,5435^{* * *}$ & 0,0080 & 1,9282 \\
\hline Óleo de soja-milho & $-4,0702^{* * *}$ & 0,0014 & 2,0733 \\
Óleo de soja-soja & $-4,2284^{* * *}$ & 0,0008 & 2,0458 \\
Óleo de soja-sorgo & $-4,2483^{* * *}$ & 0,0007 & 1,9815 \\
\hline
\end{tabular}

${ }^{* \star \star}$ Estatisticamente significante ao nível de 1\%; ** Estatisticamente significante ao nível de 5\%; * Estatisticamente significante ao nível de 10\%; ns: Estatisticamente não significativo.

Fonte: dados da pesquisa, 2020.

De acordo com os resultados expressos na Tabela 4, observou-se que as séries são estacionárias de ordem um (I(1)). Em seguida, testou-se a cointegração entre as séries, para determinar a ordem de defasagem e estimar o modelo de cointegração.

Os valores dos critérios de informação de Akaike, Schwarz e Hannan-Quinn utilizados como base de definição do número de defasagens ótimas para o teste de cointegração são apresentados na Tabela 5 .

Tabela 5 - Defasagens ótimas entre os preços dos alimentos e das commodities

\begin{tabular}{|c|c|c|c|c|c|}
\hline & \multirow{2}{*}{ Preços } & \multirow{2}{*}{ Defasagens } & \multicolumn{3}{|c|}{ Critérios de informação } \\
\hline & & & Akaike & Schwarz & Hannan-Quinn \\
\hline \multirow{3}{*}{ Arroz } & Milho & 2 & 3,91819 & 4,10799 & 3,99525 \\
\hline & Soja & 2 & 4,88891 & 5,07871 & 4,96597 \\
\hline & Sorgo & 2 & 3,71373 & 3,90353 & 3,79078 \\
\hline \multirow{3}{*}{ Feijão } & Milho & 2 & 5,01799 & 5,20779 & 5,09504 \\
\hline & Soja & 2 & 6,20041 & 6,39021 & 6,27747 \\
\hline & Sorgo & 2 & 4,89619 & 5,08599 & 4,97325 \\
\hline \multirow{3}{*}{ Leite } & Milho & 2 & 1,84641 & 2,03621 & 1,92347 \\
\hline & Soja & 3 & 2,79681 & 3,06253 & 2,90469 \\
\hline & Sorgo & 2 & 1,74199 & 1,93179 & 1,81905 \\
\hline \multirow{3}{*}{ Óleo de soja } & Milho & 2 & 1,69463 & 1,88443 & 1,77168 \\
\hline & Soja & 2 & 2,71724 & 2,90704 & 2,79429 \\
\hline & Sorgo & 2 & 1,50979 & 1,69959 & 1,58685 \\
\hline
\end{tabular}

Fonte: dados da pesquisa, 2020. 
Partindo do princípio da parcimônia, optou-se por duas defasagens ótimas para o teste de cointegração dos preços dos produtos em estudo, conforme definido pelos critérios de informação de Schwarz, com exceção da combinação de leite e soja, com três defasagens ótimas. Definido o número ótimo de defasagens, realizou-se o teste de cointegração (Tabela 6).

Tabela 6 - Teste traço para verificação de cointegração entre os preços dos alimentos em relação aos preços das commodities

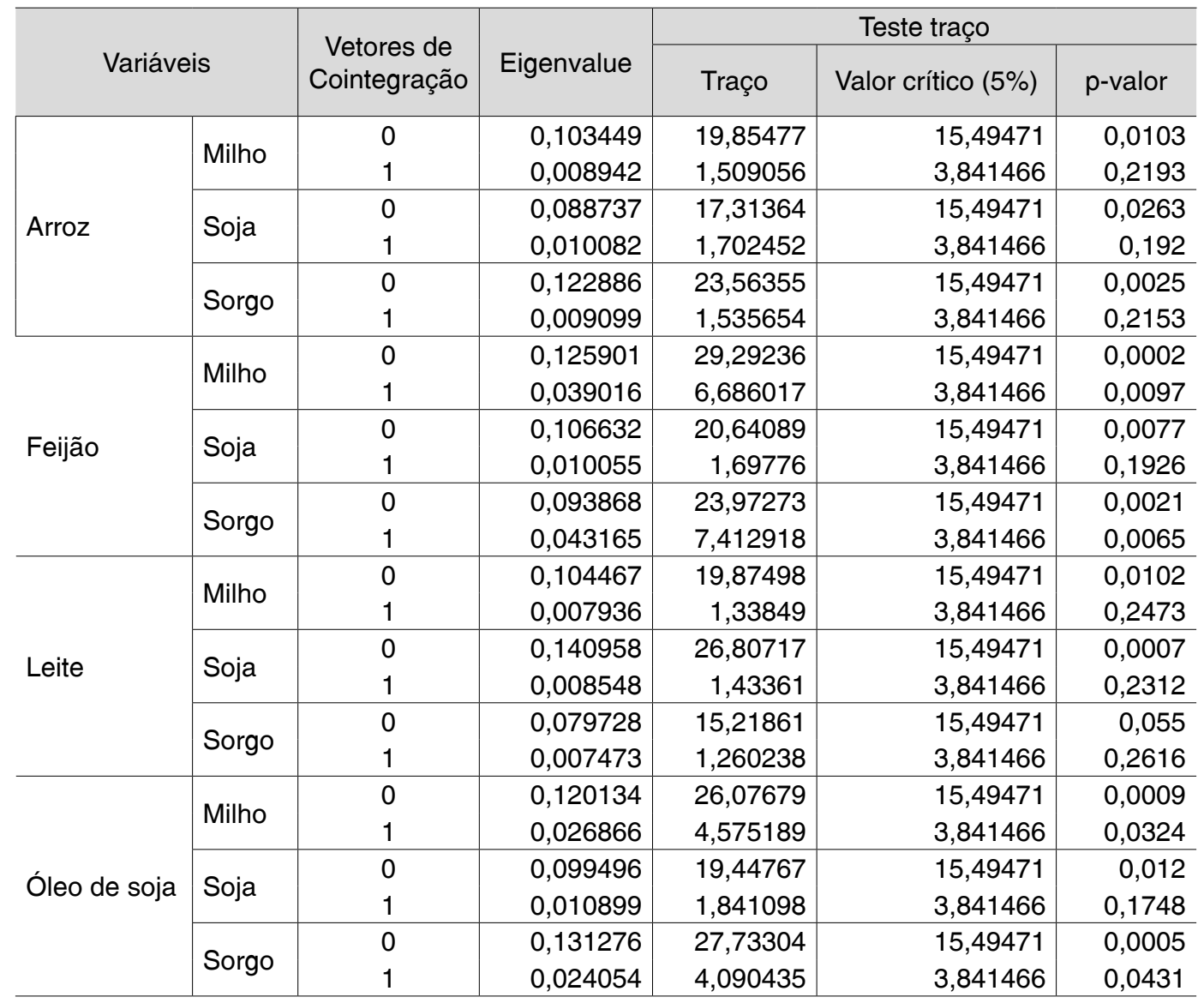

Fonte: dados da pesquisa, 2020.

Segundo os resultados apresentados na Tabela 6, verificou-se que as cointegrações entre os mercados de alimentos e os mercados de commodities em pares, os valores críticos e os valores do teste traço indicam a existência de um vetor de 
cointegração entre as séries de preços do arroz e as séries de preços do milho, do sorgo e da soja, respectivamente, considerando-se o nível de $5 \%$ de significância.

Foi identificada a existência de um vetor de cointegração entre os mercados de feijão e milho, soja e sorgo. Os resultados para o mercado de leite evidenciaram a existência de um vetor de cointegração entre as séries de preços do leite e as séries de preços do milho e da soja, ao nível de 5\% de significância.

Para analisar se há uma relação causal entre as séries estudadas, foi realizado o teste de causalidade de Granger, como pode ser visto na Tabela 7, utilizando-se das séries de retornos.

Tabela 7 - Teste de causalidade de Granger emparelhado

\begin{tabular}{l|c|c}
\hline \multicolumn{1}{c|}{ Hipótese nula } & Estatística-F & p-valor \\
\hline Milho não Granger causa arroz & 2,0155 & 0,1366 \\
Arroz não Granger causa milho & 1,2684 & 0,284 \\
Soja não Granger causa arroz & $10,1460^{* *}$ & 0,0001 \\
Arroz não Granger causa soja & 0,9126 & 0,4035 \\
Sorgo não Granger causa arroz & $3,3300^{* *}$ & 0,0382 \\
Arroz não Granger causa sorgo & 1,9515 & 0,1454 \\
Milho não Granger causa feijão & $3,9322^{* *}$ & 0,0215 \\
Feijão não Granger causa milho & 1,434 & 0,2413 \\
Soja não Granger causa feijão & $4,4474^{*}$ & 0,0132 \\
Feijão não Granger causa soja & 0,4896 & 0,6138 \\
Sorgo não Granger causa feijão & $3,2408^{* *}$ & 0,0417 \\
Feijão não Granger causa sorgo & 2,9955 & 0,0528 \\
Milho não Granger causa leite & 1,3913 & 0,2517 \\
Leite não Granger causa milho & 0,9867 & 0,375 \\
Soja não Granger causa leite & 1,4877 & 0,229 \\
Leite não Granger causa soja & $5,6419^{* * *}$ & 0,0043 \\
Sorgo não Granger causa leite & 1,0554 & 0,3504 \\
Leite não Granger causa sorgo & 1,5353 & 0,2185 \\
Milho não Granger causa óleo de soja & 1,2936 & 0,2771 \\
Óleo de soja não Granger causa milho & 1,1751 & 0,3114 \\
Soja não Granger causa óleo de soja & $4,8833^{* \cdots}$ & 0,0087 \\
Óleo de soja não Granger causa soja & 1,0523 & 0,3515 \\
Sorgo não Granger causa óleo de soja & $6,0745^{\cdots *}$ & 0,0029 \\
Óleo de soja não Granger causa sorgo & 0,3484 & 0,7063 \\
\hline
\end{tabular}

* Indica rejeição da hipótese nula ao nível de 10\%; ** Indica rejeição da hipótese nula ao nível de $5 \%$; *** Indica rejeição da hipótese nula ao nível de $1 \%$.

Fonte: dados da pesquisa, 2020. 
Como pode ser visto na Tabela 7, o teste de causalidade de Granger emparelhado indica que as alterações nos preços da soja, do milho e do sorgo, respectivamente, causam no sentido de Granger alterações nos preços do feijão. As alterações nos preços do sorgo causam no sentido de Granger alterações nos preços do óleo de soja e do arroz.

\section{Conclusões}

O objetivo da presente pesquisa foi verificar se há relacionamento de longo prazo e transmissão de preços das commodities para os preços dos alimentos no estado de Goiás. As variáveis consideradas para este estudo consistem nos preços médios mensais de arroz, feijão, leite, milho, óleo de soja, soja e sorgo, no estado de Goiás. As séries de preços das commodities consistem em milho, soja e sorgo, que são os principais produtos agropecuários produzidos em Goiás. Os demais produtos foram considerados como alimentos, visto que estes fazem parte da cesta básica.

Para analisar se existe transmissão de preços nos mercados de alimentos e commodities, aplicou-se o teste de raiz unitária de Dickey-Fuller Aumentado, para testar a estacionariedade das séries. Em seguida, para testar a existência de cointegração entre as séries, utilizaram-se o teste de cointegração de Engle e Granger e o teste de cointegração de Johansen. Para analisar se existe uma relação causal entre as séries estudadas, foi realizado o teste de causalidade de Granger.

Verificou-se que existe relacionamento de longo prazo entre os preços dos alimentos e das commodities agropecuárias, de forma que as variações nos preços das commodities podem afetar os preços dos alimentos. Com a aplicação do teste de causalidade de Granger, verificou-se que preços da soja, do milho e do sorgo, respectivamente, podem ser considerados bons previsores para o comportamento do preço do feijão no estado de Goiás. Além disso, foi possível observar que o preço do sorgo é um bom previsor para os preços do óleo de soja e do arroz.

Portanto, a preocupação sobre a volatilidade dos preços dos alimentos é pertinente. Os resultados apresentados nesta pesquisa corroboram a hipótese inicial deste trabalho, uma vez que se observou que existe uma relação de longo prazo entre os mercados de commodities e de alimentos, ou seja, o aumento da instabilidade de preços de algumas commodities agrícolas pode afetar os preços dos alimentos. 


\title{
Relación entre los precios de los alimentos y los productos agrícolas
}

\author{
Resumen
}

El objetivo del presente trabajo fue verificar si existe una relación a largo plazo y una transmisión de precios entre los mercados de productos básicos agrícolas y alimentos básicos. Para lograr los objetivos de la investigación, que implica el análisis de la transmisión de precios en los mercados de alimentos y commodities, inicialmente se intentó probar la estacionariedad de la serie en estudio. Posteriormente, se utilizó la prueba de cointegración de Engle y Granger y la prueba de cointegración de Johansen. Para analizar si existe relación causal entre las series estudiadas, se realizó la prueba de causalidad de Granger. Se encontró que existe una relación a largo plazo entre los precios de los alimentos y los productos básicos agrícolas, por lo que los cambios en los precios de los productos básicos pueden afectar los precios de los alimentos.

Palabras clave: Cointegración. Transmisión de precios. Productos básicos. Alimentos.

\section{Relationship between food prices and agricultural commodities}

\begin{abstract}
The objective of the present work was to verify if there is a long-term relationship and price transmission between the agricultural commodities and food staples markets. To achieve the objectives of the research, which involves the analysis of the transmission of prices in the food and commodities markets, initially, we tried to test the stationarity of the series under study. Subsequently, the Engle and Granger cointegration test and the Johansen cointegration test were used. To analyze whether there is a causal relationship between the series studied, the Granger causality test was performed. It was found that there is a long-term relationship between the prices of food and agricultural commodities, so that changes in commodity prices can affect food prices.
\end{abstract}

Keywords: Cointegration. Price transmission. Commodities. Foods.

\section{Referências}

ABDLAZIZ, R. A.; RAHIM, K. A.; ADAMU, P. Oil and Food Prices Co-integration Nexus for Indonesia: A Non-linear Autoregressive Distributed Lag Analysis. International Journal of Energy Economics and Policy, v. 6, n. 1, p. 82-87, 2016.

AKÉ, S. C. The Nonlinear Relation between Biofuels and Food Prices. Investigación Económica, v. 76, n. 299, p. 3-26, 2017. 
AL-MAADID, A. et al. Spillovers between food and energy prices and structural breaks. International Economics, v. 150, p. 1-18, 2017.

BUENO, R. L. S. Econometria de Séries Temporais. São Paulo: Cengage Learning, 2011.

CEBALLOS, F. et al. Grain Price and Volatility Transmission from International to Domestic Markets in Developing Countries. World Development, v. 94, p. 305-320, 2017.

DICKEY, D. A.; FULLER, W. A. Distribution of the estimators for autoregressive times series with a unit root. Journal of the American Statistical Association, v. 74, p. 427-431, 1979.

ENDERS, W.; SIKLOS, P. Cointegration and threshold adjustment. Journal of Business and Economic Statistics, v. 19, n. 2, p. 166-176, 2001.

ENGLE, R. F.; GRANGER, C. W. J. Co-integration and Error Correction: Representation, Estimation, and Testing. Econometrica, v. 55, p. 251-276, 1987.

FASANYA, I.; AKINBOWALE, S. Modelling the return and volatility spillovers of crude oil and food prices in Nigeria. Energy, v. 169, p. 186-205, 2019.

FILIP, O. et al. Food versus fuel: an updated and expanded evidence. Energy Economics, 2017.

GILBERT, C. How to Understand High Food Prices. Journal of Agricultural Economics, 2010.

GUJARATI, D. N.; PORTER, D. C. Econometria Básica. 5. ed. Porto Alegre: AMGH, 2011.

HILL, C.; GRIFFITHS, W.; JUDGE, G. Econometria. 3. ed. São Paulo: Saraiva, 2010.

JAWAD, S. et al. Asymmetric risk spillovers between oil and agricultural commodities. Energy Policy, v. 118, n. August 2017, p. 182-198, 2018.

JOHANSEN, S. Statistical analysis of cointegration vectors. Journal of Economic Dynamics and Control, v. 12, n. 2-3, p. 231-254, 1988.

NICOLA, F.; PACE, P. De; HERNANDEZ, M. A. Co-movement of major energy, agricultural, and food commodity price returns: a time-series assessment. Energy Economics, v. 57, p. 28-41, 2016.

PAL, D.; MITRA, S. K. Interdependence between crude oil and world food prices: A detrended cross correlation analysis. Physica A, v. 492, p. 1032-1044, 2018.

PAL, D.; MITRA, S. K. Time-frequency contained co-movement of crude oil and world food prices: A wavelet-based analysis. Energy Economics, 2017.

PINDYCK, R. S.; RUBINFELD, D. L. Econometria. Rio de Janeiro: Elsevier, 2004.

REZENDE, J. D. S.; OLIVEIRA NETO, O. J. de; SILVA, K. A. Volatilidade e Transmissão dos Preços Internacionais do Trigo para os Preços Domésticos do Trigo e Derivados no Brasil. Future Studies Research Journal: Trends and Strategies, v. 10, n. 1, p. 132-159, 2018.

STOCK, J. H.; WATSON, M. W. Econometria. São Paulo: Addison Wesley, 2004.

TADASSE, G. et al. Drivers and triggers of international food price spikes and volatility. Food Policy, v. 47, p. 117-128, 2014.

TORRES, D. A. P. Segurança alimentar e volatilidade de preços: uma discussão com base no projeto Foodsecure. Revista de Política Agrícola, v. 26, n. 2, p. 115-124, 2017.

WOOLDRIDGE, J. M. Introdução à econometria: uma abordagem moderna. São Paulo: Cengage Learning, 2013. 